\title{
AMBIENTE TÉRMICO Y BIENESTAR DE LOS CERDOS EN EL PERÍODO DE DESCANSO PREVIO AL SACRIFICIO
}

\author{
THERMAL ENVIRONMENT AND WELFARE OF PIGS \\ IN THE RESTING PERIOD BEFORE SLAUGHTER
}

\author{
Centurión, R.A.O. ${ }^{1 A}$; Caldara, F.R. ${ }^{1 *}$; Moi, M. ${ }^{18}$; Almeida Paz, I.C.L. ${ }^{2}$; Garcia, R.G. ${ }^{1 C}$; \\ Nääs, I.A. ${ }^{1 D}$; Alves, M.C.F. ${ }^{1 E} ;$ Zeviani, W.M. ${ }^{3}$ y Seno, L.O. ${ }^{1 F}$
}

\begin{abstract}
1Universidade Federal da Grande Dourados (UFGD). Faculdade de Ciências Agrárias. Dourados, MS.

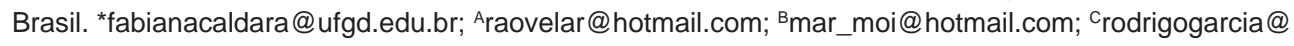
ufgd.edu.br; Direnilza@gmail.com; Emarilia.mcfa@yahoo.com.br; Fleonardoseno@ufgd.edu.br Universidade Estadual Paulista (UNESP). Faculdade de Medicina Veterinária e Zootecnia. Botucatu, SP. Brasil.ibiarapaz@fmvz.unesp.br 3Universidade Federal do Paraná. Departamento de Estatística. Curitiba, PR. Brasil.walmes@ufpr.br
\end{abstract}

\section{Palabras clave adicionales}

Aspersión. Confort térmico. Termografía infrarroja.

\section{RESUMEN}

Se evaluó la relación entre el ambiente térmico de las pocilgas de descanso en un matadero de cerdos y la temperatura superficial (TS) de los animales, así como el efecto de la pulverización intermitente de agua sobre el confort térmico. Se llevaron a cabo dos visitas a un matadero comercial, y en cada uno de ellos fueron seleccionadas tres pocilgas de descanso, una destinada al tratamiento de control (sin pulverizar durante todo el período) y dos para el tratamiento de pulverización intermitente de agua (sucesivamente 30 minutos con aspersión y 30 minutos sin aspersión). Las evaluaciones se iniciaron en el momento de la descarga de los animales, en la cual se registraron imágenes termográficas de los lotes con una cámara termográfica infrarroja. Después del alojamiento en la porqueriza se registraron imágenes termográficas y la frecuencia respiratoria (FR) de los animales cada cinco minutos durante cuatro horas. También fueron registradas la temperatura y la humedad relativa del aire y se calcularon los índices de temperatura y humedad (ITH). Considerando los valores de (ITH) se observó que los animales se mantuvieron en un estado de confort térmico sólo en el momento de su llegada al matadero. La TS y la FR de los animales mantenidos en la pocilga, sin el sistema de aspersión (control) presentaron una elevación lineal en fun-

\section{Additional KEYWORDS}

Infrared thermography. Spraying. Thermal comfort.

ción de la hora del día, o sea, conforme al aumento de la temperatura ambiental. En el tratamiento con aspersión intermitente de agua se observó un comportamiento sinusoidal del TS y FR, entretanto las mismas se mantuvieron siempre inferiores al tratamiento control, incluso en los momentos que los rociadores de agua se encontraban desconectados. El tiempo de aspersión necesario para la reducción y estabilización de la TS de los animales estuvo condicionado al binomio temperatura-humedad relativa del aire, siendo estimado en 25 minutos en los horarios de mayor ITH. La temperatura ambiente máxima durante las evaluaciones fue de $28,4{ }^{\circ} \mathrm{C}$.

\section{SUMMARY}

The research aimed to evaluate the relationship between the thermal environment of the rest pens in a pig slaughterhouse and the surface temperature (ST) of the animals as well as the effect of intermittent water spraying on thermal comfort. There were two visits to slaughter industrial being in each selected three pens of rest, one for control treatment (no spray throughout the period) and two for the treatment of intermittent spraying water (30 minutes intervals with constant spray followed by 30 minutes then turned off).

Recibido: 12-11-12. Aceptado: 9-4-13. 
Assessments began at the time of discharging animals in which are taken the thermographic images from lots using an infrared thermographic camera. After housing in the pens, were registered the thermographic images and the respiratory frequency (RF) every five minutes for four hours. Were evaluated the temperature, relative humidity and temperature-humidity index (THI). Considering the values of THI was observed that the animals remained in a condition of comfort only at the time of his arrival at the slaughterhouse. The ST and RF of the animals kept in the pens without sprinkler system (control) showed a linear increase in function of time of day, with increasing temperature. The treatment with intermittent sprinkling of water was observed oscillatory behavior of the ST and RF, however it was always lower than the control treatment, even in times when the water sprinklers were turned off. The time required of spray to reduction and stabilization of the ST of the animals was limited to the combination of temperature and relative humidity, being estimated at 25 minutes at times of higher THI. The maximum environmental temperature during evaluation was $28.4^{\circ} \mathrm{C}$.

\section{INTRODUCCIÓN}

La preocupación por la producción de alimentos está cada vez más centrada en la calidad del producto final y sus implicaciones sobre el medio ambiente y el bienestar animal (Ferreira, 2000). Son notables las variaciones del tiempo y el clima en Brasil (el Amazonas es caliente y húmedo y el nordeste semiárido); las estaciones seca y lluviosa en la Meseta Central se suceden y en el Sur las lluvias son regulares) y se registra influencia del clima sobre el bienestar animal, la productividad y la calidad de la carne (Ferreira, 2000).

El estrés térmico, una de las principales causas de pérdidas de producción en climas cálidos, se puede minimizar con el uso adecuado de equipos y procesos (Lin et al., 2006; Barbari et al., 2007) en las instalaciones zootécnicas, para controlar principalmente las temperaturas ambientales, condiciones de humedad y ventilación.

Carvalho et al. (2004) verificaron que la nebulización y ventilación forzada mejoran el confort, medido a través de la temperatura de la piel, en cerdos en fase de terminación, registrando mejor comportamiento social, mayor tranquilidad y casi ninguna competición por los comederos y bebederos colectivos en las horas más calurosas del día.

El manejo previo al sacrificio (embarque, transporte, desembarque) predispone a los cerdos a numerosos factores de estrés que determinan la aceleración del metabolismo y temperatura corporal elevada. El periodo de descanso en el matadero es una importante práctica de manejo que está siendo utilizada para posibilitar la recuperación de los animales del estrés físico y emocional ocasionado por el transporte, lo que repercute en una mejora del producto final obtenido.

La termografía mide la energía de radiación infrarroja de los cuerpos permitiendo estudiar patrones de distribución de temperatura a través del intercambio de calor y obtener imágenes térmicas. La medición se puede aplicar a cualquier sistema, objeto o proceso donde hay un intercambio térmico con el medio ambiente. El uso de imágenes termográficas es útil en los estudios de termorregulación (Knizkova et al., 2007) debido a que la cuantificación de la temperatura de la superficie de los animales es crucial para estimar el ambiente térmico de alojamiento de forma más precisa (Yahav et $a l ., 2004)$. La estimación más exacta de la transferencia de calor, da una mejor comprensión de la eficiencia energética del animal (Cangar et al., 2008).

El objetivo de este estudio fue evaluar la relación entre el ambiente térmico de las pocilgas de descanso en un matadero de cerdos y la temperatura superficial de los animales y el efecto de los sistemas de pulverización intermitente de agua sobre el confort térmico.

\section{MATERIAL Y MÉTODOS}

La investigación se llevó a cabo entre 


\section{BIENESTAR DE LOS CERDOS EN EL PERÍODO DE DESCANSO}

marzo y abril de 2011 en un matadero comercial, situado en Dourados, MS, Brazil, con el Servicio de Inspección Federal (SIF) y con una capacidad de sacrificio de 2300 cabezas por día, lo que representa aproximadamente el $50 \%$ del sacrificio diario en el Estado de Mato Grosso do Sul. La mayoría de los sistemas de producción porcina integrados en la empresa está ubicada en el mismo municipio o en el área circundante a una distancia de $100 \mathrm{~km}$ de la planta faenadora.

La altitud de la ciudad es de $430 \mathrm{~m}$, latitud $22^{\circ} 13^{\prime} \mathrm{S}$ y longitud $54^{\circ} 48^{\prime} \mathrm{W}$. El clima, según la clasificación de Köppen, es Aw (clima tropical con lluvias en verano y seco en invierno).

Para la conducción de la investigación, en abril se llevaron a cabo dos visitas al matadero comercial y en cada una de ellas fueron seleccionadas tres pocilgas de descanso, una destinada al tratamiento de control (sin pulverizar durante todo el período de permanencia de los animales) y dos para el tratamiento intermitente de pulverización de agua (intervalos constantes de 30 minutos con aspersión seguidos por 30 minutos sin aspersión). Todos los animales fueron sometidos a un proceso anterior similar, en cuanto al tiempo, ayuno sólido, distancia, duración y horario de transporte, horario de descarga y conducción de animales para las pocilgas de descanso dentro del matadero, siendo adoptado el procedimiento estándar empleado por la empresa.

Los datos ambientales de temperatura máxima (Tmax), temperatura mínima (Tmin), humedad relativa máxima (HRmax) y la humedad relativa mínima (HRmin) recogidos en la Estación Meteorológica de la Universidade Federal da Grande Dourados, en dos días de evaluación fueron, respectivamente, $34,7^{\circ} \mathrm{C}$ e $33,6{ }^{\circ} \mathrm{C}$ (Tmax), 22,5 e $21,6{ }^{\circ} \mathrm{C}$ (Tmin), 85,5 e 68,4\% (HRmax) e 37,6 e 34,3 $\%$ (HR min). No hubo precipitaciones en los días de evaluación.

Los animales, machos castrados, con un peso promedio de $120 \mathrm{~kg}$, fueron sometidos a un tiempo de ayuno previo al transporte de aproximadamente seis horas. Después del período de ayuno, los animales más pesados por corral fueron seleccionados y dirigidos para el embarque, con el uso de lonas para clasificación. La conducción se realizaba con la ayuda de cascabeles (botella de plástico con piedras dentro) y láminas de manejo, habiendo de esa forma una mezcla de animales procedentes de distintos corrales a partir de ese momento.

Los animales fueron embarcados utilizándose rampas elevadizas hasta la carrocería del camión y luego fueron mojados al término del embarque. Los cerdos fueron transportados al matadero en camiones (camión doble eje- con 16 divisores y tracto camión- con 24 divisores) equipados con carrocería de aluminio de dos plantas con una densidad de transporte de $0,40 \mathrm{~m}^{2}$ por cada100 kg peso vivo. La carga y el transporte se realizaron en la mañana temprano. El transporte tuvo una duración media de 2 horas.

Al llegar al matadero, los animales fueron desembarcados, bañados con agua, tatuados con pintura no tóxica y encaminados a la pocilga de descanso (boxes de $42 \mathrm{~m}^{2}$, con 10 bebederos tipo chupeta y capacidad para 65 animales), donde permanecieron durante unas seis horas hasta que se enviaron a la sala de sacrificio.

En el momento de la descarga fueron registradas las imágenes termográficas de los lotes, primero de los animales que estaban en la parte superior de la carrocería y después de los que estaban en la parte inferior. El promedio de animales por imagen fue de 50 cerdos.

En las pocilgas de descanso, las imágenes termográficas se registraron cada cinco minutos durante 4 horas hasta la salida para la matanza, tomando cuatro imágenes por pocilga. También se registraron imágenes termográficas de la cubierta del recinto a intervalos de 30 minutos.

El registro de imágenes termográficas se realizó usando el equipo generador de imágenes térmico Testo ${ }^{\circledR} 876-1$ kit profesional 
con una precisión de $\pm 0,1^{\circ} \mathrm{C}$ y 7,5 a $13 \mathrm{mM}$ rango del espectro infrarrojo y por medio de un software específico para este equipo, la lectura en el espectro de color se convierte en la temperatura superficial. El coeficiente de emisividad de 0,96 fue utilizado para toda la superficie del cuerpo del animal. La media y desviación estándar de la temperatura de la superficie del cuerpo, se calculó utilizando la temperatura de puntos seleccionados al azar, con el fin de representar la superficie total del cuerpo del animal, o mediante la selección de áreas que cubran el mayor número de animales y toda su superficie corporal, para la construcción de un histograma de temperatura. Se consideró para la evaluación, la media de la temperatura de los puntos del conjunto de animales en cada imagen. Al mismo tiempo, se midió la frecuencia respiratoria de dos animales por pocilga, seleccionados aleatoriamente mediante observación del número de movimientos respiratorios por minuto.

En los días de evaluación se registraron los datos ambientales (temperatura y humedad relativa) en las pocilgas de descanso de cerdos utilizándose un termohidrómetro con registro cada 30 minutos.

Utilizando los valores medios de temperatura y humedad relativa del aire se calcularon la temperatura de bulbo seco (TBS) y el bulbo húmedo (TBH) de aire a través del programa Psicrom ${ }^{\circledR}$ (Roriz, 2003) y, posteriormente, los índices de temperatura y humedad (ITH) según la ecuación (Roller y Goldman, 1969):

$$
\text { ITH= 0,45 Tbu+1,35 Tbs+32 }
$$

Teniendo en cuenta que la temperatura de la superficie es una variable continua y su valor medio puede ser una función del tratamiento (con y sin aspersión intermitente) y hora del día (que lleva en consideración alteraciones de la temperatura ambiente) se utilizaron modelos lineales aditivos generalizados para modelar el valor esperado de la temperatura superficial. Para adap- tarse a este modelo se utilizó la función gam del paquete mgcv (Wood, 2000, 2003, 2004, 2011). Ambos se ajustaron a cuatro modelos: 1) o nulo, donde la temperatura es constante, no depende del tratamiento (con o sin aspersión de agua), o de la hora del día; 2) en el que la temperatura depende de una función leve con relación la hora del día, pero no depende del tratamiento; 3) que considera el efecto aditivo del tratamiento y la hora del día (sin interacción), y 4) que considera el efecto completo de la interacción entre el tratamiento y la hora del día sobre la temperatura superficial de los animales. Este último fue el modelo seleccionado, explicando en un $84 \%$ la varianza en los datos $\left(R^{2}=0,84\right)$.

Los datos de la frecuencia respiratoria fueron sometidos a análisis de varianza y las medias se compararon mediante la prueba de Tukey. Las medias de frecuencia respiratoria, la temperatura superficial, la temperatura del aire y la temperatura del tejado se sometieron a análisis de correlación de Pearson mediante el programa estadístico ASSISTAT (2012). En todos los casos el nivel de significación se fijó en $\mathrm{p}<0,05$.

\section{RESULTADOSYDISCUSIÓN}

El ambiente térmico abarca, entre otros factores, los efectos de la radiación solar, temperatura ambiental, humedad relativa del aire y velocidad del viento (Falco, 1997; Baêta y Souza, 2010). La combinación de temperatura y humedad relativa es uno de los principales condicionantes del confort térmico y el funcionamiento general de los procesos fisiológicos. Considerando conjuntamente estos factores climáticos para la determinación del índice de temperatura y humedad (ITH), se observó que los animales permanecieron en condiciones de confort sólo a su llegada al matadero (ITH $\leq 74)$, y posteriormente se alcanzó un estado de alerta (ITH $\geq 75)$ e incluso una condición peligrosa (79 a 83) (Barbari et al., 2007) (tabla I). 


\section{BIENESTAR DE LOS CERDOS EN EL PERÍODO DE DESCANSO}

Analizando las imágenes termográficas cuando los animales llegaron al matadero, se constató que los cerdos transportados en la planta superior de la carrocería mostraron mayor temperatura superficial, lo que demuestra el efecto de la radiación solar directa sobre los animales cuando los animales son transportados en un camión sin techo que los proteja.

La temperatura superficial de los animales que permanecieron en la pocilga sin aspersor (control) mostró un aumento lineal en función de la hora del día, es decir, conforme al aumento de la temperatura ambiental.

Considerando el amoldado de la temperatura corporal por el modelo lineal aditivo generalizado completo, se observa que el valor esperado para la temperatura superficial (TS) es igual entre los tratamientos en el momento inmediatamente anterior a la activación del aspersor (9:00 am). Esta homogenización puede deberse al hecho que todos los animales se humedecieron inmediatamente después del desembarque

Tabla I. Temperatura $($ Tar $)\left({ }^{\circ} \mathrm{C}\right)$ y humedad relativa (\% $\mathrm{HR}$ ) del aire, medidas en las pocilgas durante el periodo de descanso antes de la matanza e índices de temperatura y humedad (ITH) calculados. (Air temperature $(\operatorname{Tar})\left({ }^{\circ} \mathrm{C}\right)$ and relative humidity $(\% \mathrm{RH})$, measured in the pens during the resting period before slaughter and calculated temperature and humidity index $(\mathrm{THI})$ ).

\begin{tabular}{lccc}
\hline Tiempo & $\operatorname{Tar}\left({ }^{\circ} \mathrm{C}\right)$ & $\mathrm{HR}(\%)$ & $\mathrm{TH}$ \\
\hline Desembarque & 24,1 & 78,4 & 74,1 \\
30 minutos & 25,4 & 71,0 & 75,9 \\
60 minutos & 26,7 & 69,3 & 78,1 \\
90 minutos & 26,6 & 73,3 & 78,2 \\
120 minutos & 27,2 & 68,4 & 78,9 \\
150 minutos & 27,9 & 66,4 & 80,0 \\
180 minutos & 28,4 & 58,8 & 80,3 \\
210 minutos & 27,7 & 59,6 & 79,1 \\
\hline
\end{tabular}

*Desde la llegada al matadero. antes de ser alojados en las pocilgas de descanso, presentando todos las mismas condiciones iniciales. En la pocilga sin aspersión la TS aumentó de manera lineal de 9:00 a 13:00 horas. La temperatura superficial de los animales mantenidos bajo aspersión intermitente mostraron un comportamiento sinusoidal disminuyendo con la aspersión y elevándose en los intervalos cuando fueron interrumpidos; con una menor amplitud de la variación en las primeras horas del día. Sin embargo, en ningún momento llegó a alcanzar la misma temperatura superficial de los animales en el tratamiento de control (figura 1).

Cuando la temperatura corporal aumenta se produce vasodilatación, con aumento del flujo sanguíneo en los capilares de la epidermis; lo que incrementa la temperatura superficial y la transferencia del calor para el ambiente. La elevación de la temperatura de la piel, aumenta el gradiente térmico entre la piel y el ambiente, resultando en una mayor pérdida de calor por irradiación y convección. Si la vasodilatación es insuficiente para mantener la temperatura normal, entonces aumenta el enfriamiento por evaporación por sudoración, por jadeo, o ambos. El enfriamiento por evaporación es el proceso predominante cuando la temperatura ambiente supera a la temperatura de la piel (Robinson, 2004). Los cerdos son especialmente susceptibles a elevadas temperaturas debido a su limitada capacidad de sudoración (Kunavongkrit et al., 2005) ya que poseen escasas glándulas sudoríparas (Dyce et al., 1997). En consecuencia, en situaciones de estrés térmico aumenta la frecuencia respiratoria para acentuar la pérdida de calor por evaporación compensando la pérdida mínima que se produce por sudoración.

Según Curtis (1983), la temperatura de la piel es la primera señal de respuesta de estrés térmico en los mamíferos y los cerdos, comenzando el jadeo cuando la temperatura de la piel se eleva por encima de $35^{\circ} \mathrm{C}$. Sin embargo, aunque hasta aproximadamen- 


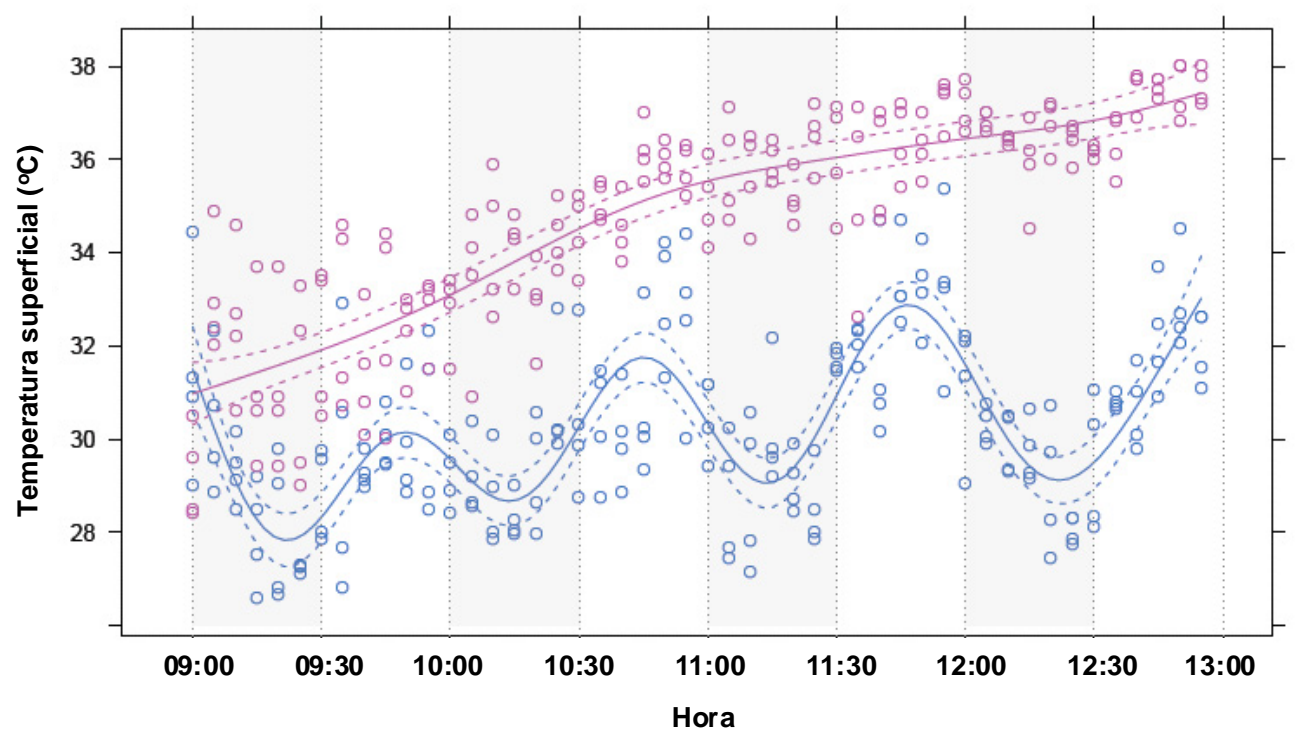

Figura 1. Valores observados y esperados de temperatura superficial de los cerdos en las pocilgas de descanso en función a la hora del día. Rectángulos grises indican períodos con sistema de aspersión encendido. Líneas continuas representan los valores esperados y las líneas discontinuas delimitan el intervalo de confianza del $95 \%$ para el valor esperado. (Observed and expected values of surface temperature of the pigs in resting pens according to the time of day. Gray rectangles indicate periods with sprinkler system operating. Solid lines represent the expected values and the dashed lines delimit the confidence interval of $95 \%$ for the expected value).

te 120 minutos después de la llegada al matadero, la TS de los cerdos del grupo control no llegó a $35^{\circ} \mathrm{C}$, estos habían aumentado la frecuencia respiratoria $(\mathrm{p}<0,05)$ con anterioridad (figura 2).

La frecuencia respiratoria de los cerdos sin pulverización de agua fue influida $(p<0,05)$ por la hora del día, o por combinación de temperatura y humedad relativa del aire, alcanzando valores superiores a 97 movimientos por minuto cuando el ITH fue mayor que 76, mientras que en los animales bajo aspersión de agua intermitente no superó en ningún momento los 75 movimientos por minuto, incluso cuando los aspersores de agua estaban parados.

Se observó una correlación alta y positiva entre la temperatura de la superficie de la piel y la frecuencia respiratoria de los animales. Las correlaciones (tabla II) entre parámetros fisiológicos y condiciones ambientales (temperatura del aire y el techo), también positivas, demuestran la influencia del ambiente sobre el estrés térmico.

Yan y Yamamoto (2000) evaluaron la pérdida de calor por evaporación, la temperatura rectal y frecuencia respiratoria en los cerdos expuestos a temperaturas ambientales de $10,15,20,25,30$ y $35^{\circ} \mathrm{C}$ durante 8 horas por día y constataron un aumento de la temperatura rectal, frecuencia respiratoria y mayor pérdida de calor por evaporación cuando la temperatura ambiente alcanzó $30{ }^{\circ} \mathrm{C}$ o al superar este umbral. Kiefer et al. (2010) observaron que la tasa respiratoria de los cerdos en terminación aumentó de 41 


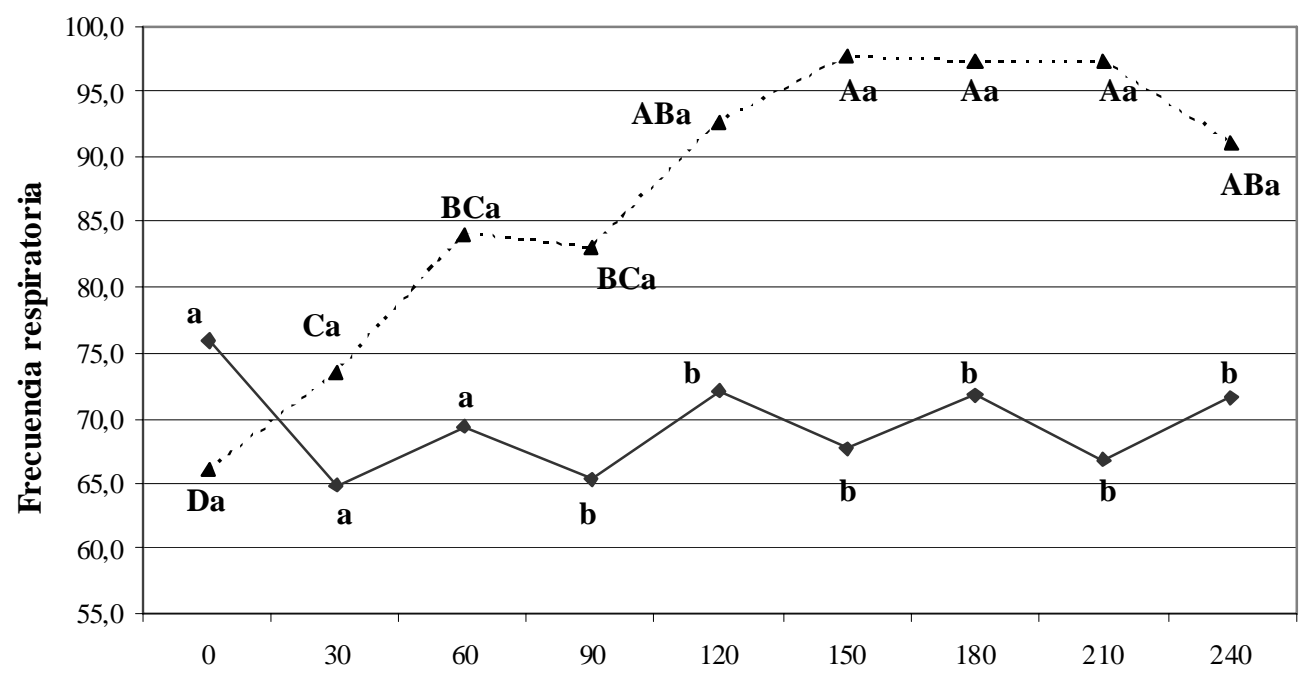

Tiempo transcurrido después de la llegada al matadero (minutos)

$\longrightarrow$ Con aspersión $\cdots . \cdot$ Sin aspersión

Las letras mayúsculas distintas dentro del mismo tratamiento a lo largo del tiempo, indican diferencias por la prueba de Tukey $(\mathrm{p}<0,05)$. Las letras minúsculas distintas entre tratamientos en cada momento indican diferencias por la prueba $\mathrm{F}(\mathrm{p}<0,05)$.

Figura 2. Frecuencia respiratoria de los cerdos sometidos a 240 minutos de descanso antes de la matanza en pocilgas con y sin (control) sistema intermitente de aspersión de agua durante todo el periodo. (Respiratory rate of pigs subjected to 240 minutes of rest before slaughter in pens with and without (control) intermittent water spraying.

Tabla II. Correlaciones entre parámetros ambientales y fisiológicos de cerdos sometidos a cuatro horas de descanso antes del sacrificio. (Correlations between environmental and physiological parameters of pigs subjected to four hours of rest before slaughter).

\begin{tabular}{lccc}
\hline & FR & TS & TT \\
\hline TS & $0,919^{* *}$ & - & \\
TT & $0,889^{* *}$ & $0,958^{*}$ & - \\
TA & $0,918^{* *}$ & $0,908^{* *}$ & $0,942^{* *}$ \\
\hline
\end{tabular}

$\mathrm{FR}=$ frecuencia respiratoria; $\mathrm{TS}=$ temperatura de la superfície corporal ; TT= temperatura del techo; $\mathrm{TA}=$ temperatura ambiente.

${ }^{*} p<0,05 ;{ }^{* *} p<0,01$ a 93 movimientos respiratorios por minuto cuando la temperatura aumentó de 21 a $32^{\circ} \mathrm{C}$.

En el presente estudio, incluso presentando un comportamiento sinusoidal, la variación de la temperatura de los animales mantenidos bajo sistema intermitente de aspersión estuvo condicionado a la temperatura ambiental. Se observó que 60 minutos después de alojar a los animales en la pocilga de descanso (10:00), se necesitaron apenas 10 minutos de aspersión de agua para que su temperatura superficial se redujera y estabilizase en torno a los $28^{\circ} \mathrm{C}$. Sin embargo, después de 180 minutos de la llegada (12:00), fueron necesarios 25 minutos de aspersión para que la temperatura 


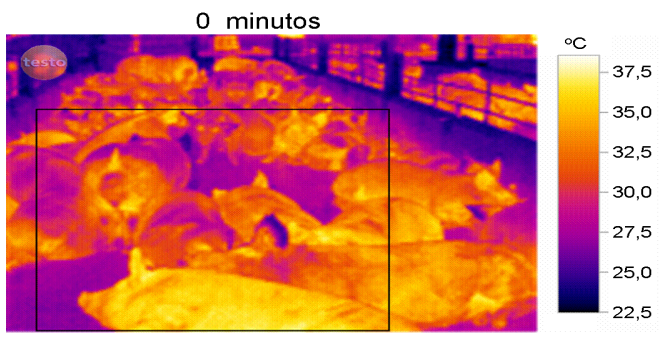

5 minutos

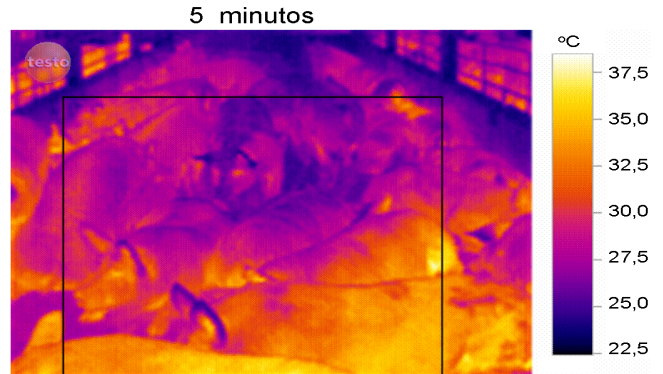

10 minutos

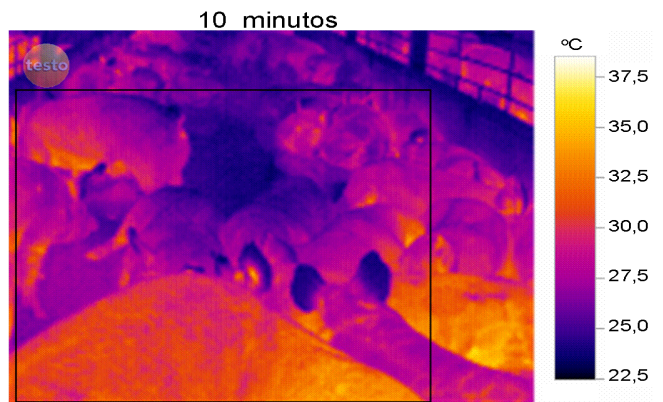

15 minutos

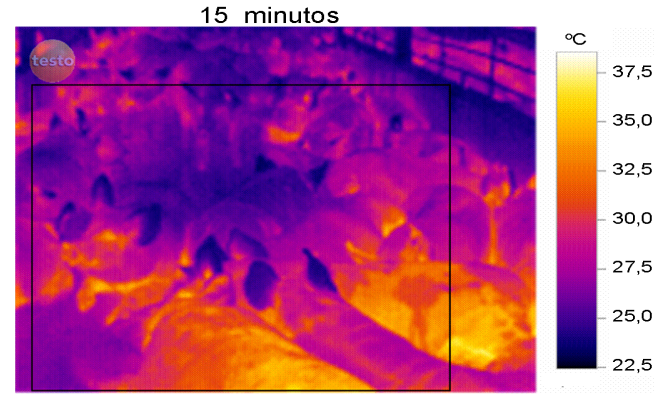

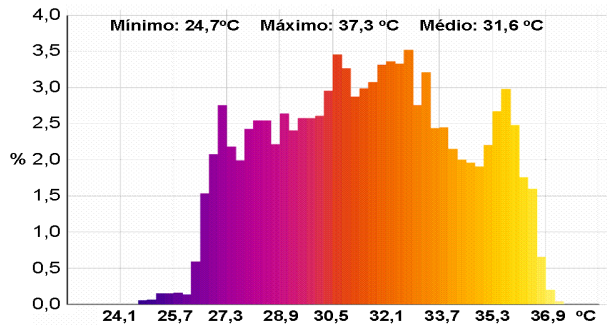
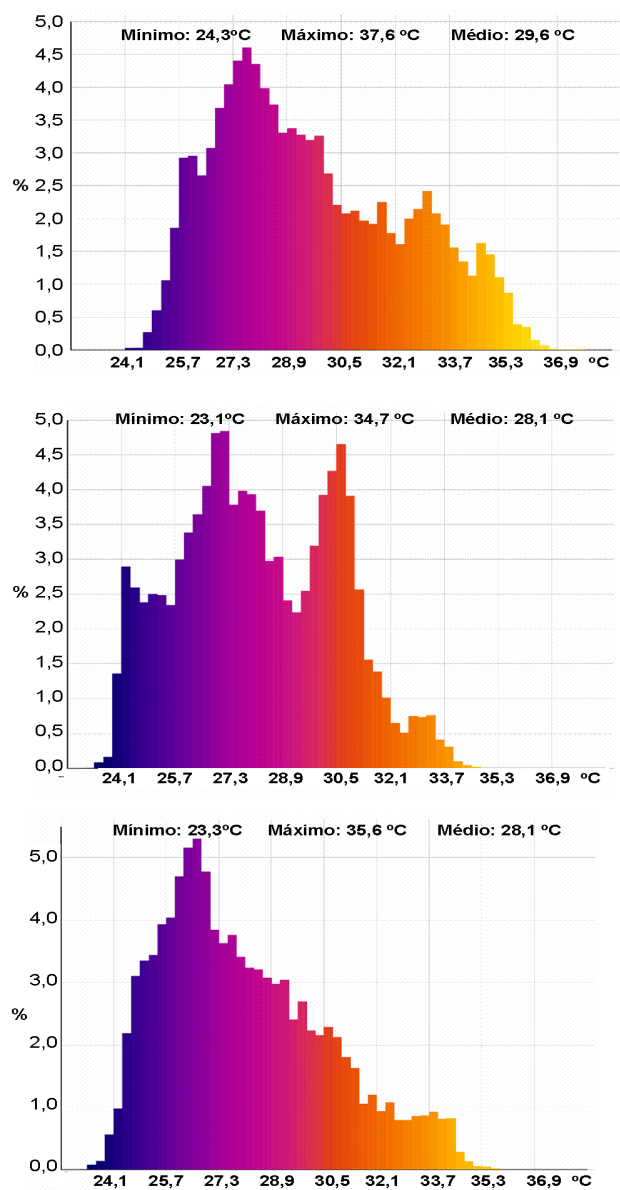

Figura 3. Imágenes termográficas de cerdos registradas en pocilgas de descanso, 60 minutos después de que los animales lleguen al matadero, en los minutos 0, 5, 10 y 15 después de la activación de los aspersores (a la izquierda). Histograma de la temperatura superficial calculado en base a la zona señalada de la imagen (a la derecha). (Thermographic images of the pigs recorded in rest pens, 60 minutes after the animals arrive in the slaughterhouse, in minutes 0 , 5,10 and 15 after activation of the sprinklers (left). Histogram of the surface temperature calculated based on the area marked on the image (right).

Archivos de zootecnia vol. 63, núm. 242, p. 246. 


\section{BIENESTAR DE LOS CERDOS EN EL PERÍODO DE DESCANSO}
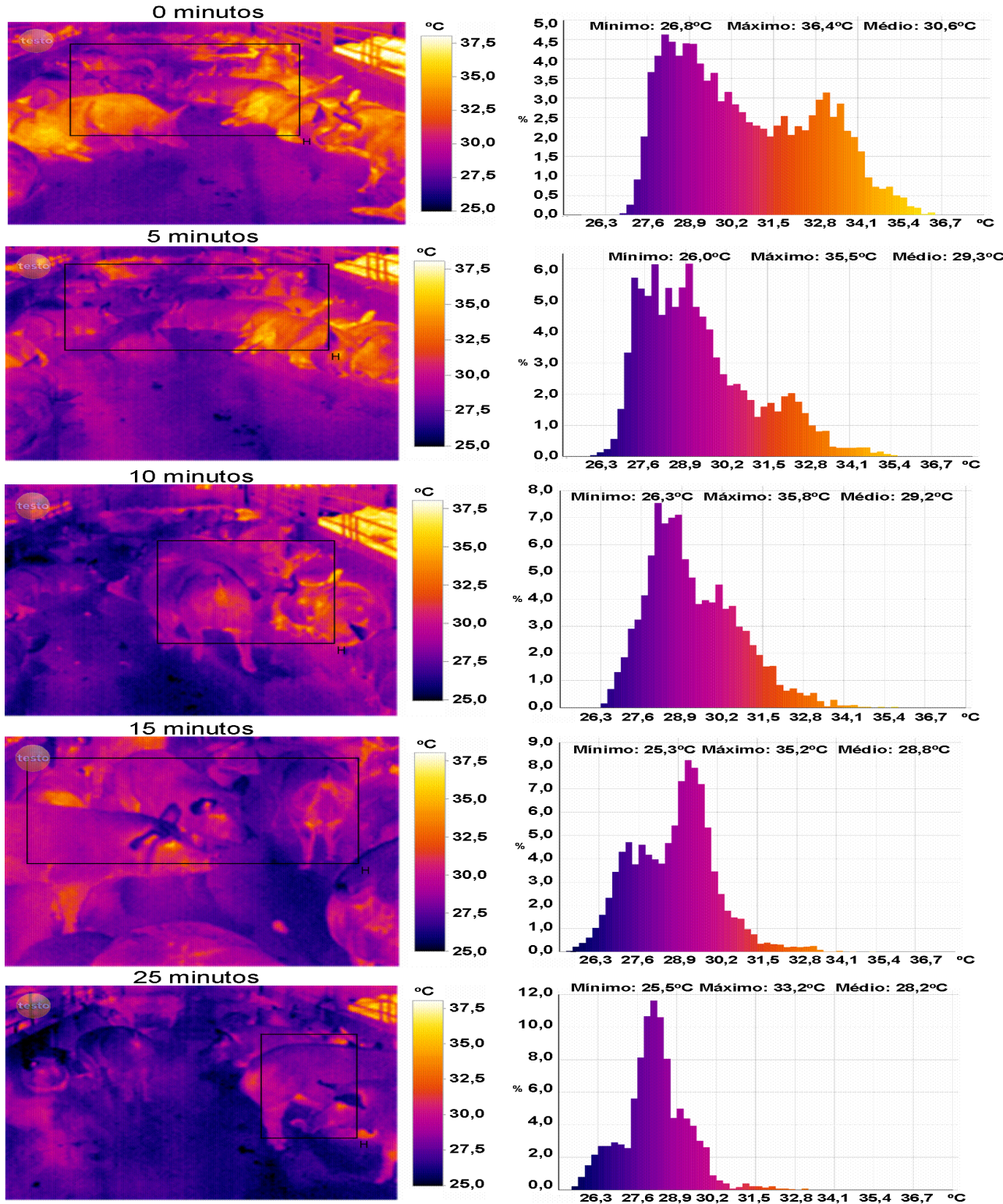

Figura 4. Imágenes termográficas de cerdos registradas en pocilgas de descanso, 180 minutos después de que los animales lleguen al matadero, a los 0, 5, 10, 15 y 25 minutos después de la activación de los aspersores (izquierda). Histograma de la temperatura superficial calculado en base a la zona señalada de la imagen (a la derecha). (Thermographic images of the pigs recorded in rest pens, 180 minutes after the animals arrives in the slaughterhouse, in minutes $0,5,10,15$ and 25 after activation of the sprinklers (left). Histogram of the surface temperature calculated based on the area marked on the image (right)).

Archivos de zootecnia vol. 63, núm. 242, p. 247. 


\section{CENTURIÓN, CALDARA, MOI, ALMEIDA PAZ, GARCIA, NÄÄS, ALVES, ZEVIANIY SENO}

superficial de los mismos llegara al mismo valor (figuras 3 y 4 ).

Mediante el uso de un sistema de ventilación con nebulización, Carvalho et al. (2004) observaron una reducción en la temperatura de la piel de los cerdos en la fase de terminación de 35,39 a $31,64{ }^{\circ} \mathrm{C}$.

En condiciones de temperatura entre 10 y $30^{\circ} \mathrm{C}$ y de humedad relativa del aire inferior a $80 \%$, la aspersión en el área de espera es deseable. Por debajo de $5^{\circ} \mathrm{C}$, la aspersión puede hacer que el animal tenga frío, tiemble y sufra espasmos, lo que puede repercutir en la aparición de una carne más oscura (DFD) (Homer y Matthews, 1998; Knowles et al., 1998). Es comúnmente aceptado que el sistema de aspersión debe ser intermitente y con una duración máxima de 30 minutos para conseguir el mayor efecto de enfriamiento y reducir la actividad y la agresividad (Weeding et al., 1993; Jones, 1999), pero no hay acuerdo en cuanto al tiempo y el número de aplicaciones. En este trabajo, el tiempo estuvo condicionado al binomio de temperatura y humedad relativa del aire, siendo estimado en 25 minutos en las horas pico ITH. Sin embargo, la temperatura ambiental máxima observada durante las evaluaciones fue de $28,4{ }^{\circ} \mathrm{C}$, pudiendo así haber una necesidad de mayor tiempo de aspersión cuando la temperatura del aire

\section{BIBLIOGRAFÍA}

ASSISTAT. 2012. Assistat Sofware (Versão 7.6 beta). Universidade Federal de Campina Grande (UFCG). Campina Grande, PB.

Baêta, F.C. e Souza, C.F. 2010. Ambiência em edificações rurais: conforto animal. 2. ${ }^{a}$ ed. Editora UFV. Viçosa. 269 pp.

Barbari, M.; Bianchi, M. and Guerri, F.S. 2007. Preliminary analysis of different cooling systems of sows in farrowing room. J Agric Eng, 1: 915.

Cangar, Ö.; Aerts, J.M.; Buyse, J. and Berckmans, D. 2008. Spatial distribution of surface temperatures and heat loss in broiler chickens. Livestock Environment VIII. ASABE. Cataratas fuese superior a este valor.

Las imágenes termográficas pusieron de manifiesto que la disipación de calor es diferente según regiones del cuerpo, siendo el intercambio de calor con el ambiente más intenso en la región de la cabeza y orejas, seguido por otras regiones del cuerpo y las piernas.

Evaluando la temperatura superficial del tejado de las pocilgas de descanso, se deduce que el material utilizado para cubrir, debe transmir menor carga de calor al interior del edificio. Se observó que la temperatura media de la superficie del techo había alcanzado un aumento lineal en función de la hora del día, alcanzando valores de aproximadamente $48^{\circ} \mathrm{C}$ a las 13:00 h.

\section{CONCLUSIÓN}

Las condiciones ambientales en las pocilgas de descanso tienen efecto directo sobre los parámetros fisiológicos de los cerdos en este periodo. La aspersión intermitente de agua, con intervalos de $30 \mathrm{minu}$ tos durante el período de descanso antes de la matanza, es eficiente en la reducción de la temperatura superficial y el mantenimiento de la frecuencia respiratoria. La planificación para el correcto funcionamiento de los aspersores de agua se condiciona al binomio temperatura y humedad relativa del aire. do Iguaçu. Brasil. 8 pp.

Carvalho, L.E.; Oliveira, S.M.P. e Turco, S.H.N. 2004. Utilização da nebulização e ventilação forçada sobre o desempenho e a temperatura da pele de suínos na fase de terminação. Rev Bras Zootecn, 33: 1486-1494.

Curtis, S.E. 1983. Environmental management in animal agriculture. 2. ${ }^{\mathrm{a}}$ ed. Ed. Iowa State University Press. Ames, lowa. 409 pp.

Dyce, K.M.; Sack, W.O. e Wensing, C.J.G. 1997. Anatomia veterinária. 2. ${ }^{\text {a }}$ ed. Guanabara Koogan. Rio de Janeiro. 663 pp.

Falco, J.E. 1997. Bioclimatologia animal. UFLA. Lavras. 57 pp. 


\section{BIENESTAR DE LOS CERDOS EN EL PERÍODO DE DESCANSO}

Ferreira, R.A. 2000. Efeitos do clima sobre a nutrição de suínos. http://www.cnpsa.embrapa.br/ abraves-sc/pdf/Memorias2000/1_RonyFe rreira.pdf (02/07/2010).

Homer, D.B. and Matthews, K.R. 1998. A repeat national survey of muscle $\mathrm{pH}$ values in commercial pig carcasses. Meat Sci, 49: 425433.

Jones, T. 1999. An investigation and assessment of the handling-systems of twelve abattoirs in Great Britain and four in Italy. Improved handling systems for pigs at slaughter. $\mathrm{PhD}$ thesis. Royal Veterinary College. University of London. UK.

Kiefer, C.; Moura, M.S.; Silva, E.A.; Santos, A.P.; Silva, C.M.; Luz, M.F.; Nantes, C.L.; Meignen, B.C.G.; Sanches, J.F. e Carrijo, A.S. 2010. Respostas de suínos em terminação mantidos em diferentes ambientes térmicos. Rev Bras Saúde Prod Anim, 11: 496-504.

Knizkova, I.; Kunc, P.; Gurdil, G.A.K.; Pinar, Y. and Selvi, K.C. 2007. Applications of infrared thermography in animal production. J Fac Agric, 22: 329-336.

Knowles, T.G.; Brown, S.N.; Edwards, J.E. and Warris, P.D. 1998. Ambient temperature below which pigs should not be continuously showed in lairage. Vet Rec, 143: 576-578.

Kunavongkrit, A.; Suriyasomboon, A.; Lundeheim, N.; Heard, T.W. and Einarsson, S. 2005. Management and sperm production of boars under differing environmental conditions. Theriogenology, 63: 657-667.

Lin, H.; Hao, H.C.; Buyse, J. and Decuypere, E. 2006. Strategies for preventing heat stress in poultry. Worlds Poultry Sci J, 62: 71-95.

Robinson, N.E. 2004. Homeostase- Termorre- gulação. In: Cunningham, J.G. Tratado de fisiologia veterinária. 3. ${ }^{\mathrm{a}}$ ed. Guanabara Koogan. Rio de Janeiro. pp. 550-560.

Roller, W.L. and Goldman, R.F. 1969. Response of swine to acute heat exposure. Transactions ASABE, 12: 164-169.

Roriz, M. 2003. Psicrom 1.0 - Relações Psicométricas. Universidade Federal de São Carlos. Departamento de Engenharia Civil. Programa de Pós-Graduação em Construção Civil. São Carlos.

Weeding, C.M.; Guise, H.J. and Penny, R.H.C. 1993. Factors influencing the welfare and carcass and meat quality of pigs: the use of water sprays in lairage. Anim Prod, 56: 393397.

Wood, S.N. 2000. Modelling and smoothing parameter estimation with multiple quadratic penalties. J Roy Stat Soc (B), 62: 413-428.

Wood, S.N. 2003. Thin-plate regression splines. J Roy Stat Soc (B) 65: 95-114.

Wood, S.N. 2004. Stable and efficient multiple smoothing parameter estimation for generalized additive models. J Am Stat Assoc, 99: 673-686.

Wood, S.N. 2011. Fast stable restricted maximum likelihood and marginal likelihood estimation of semiparametric generalized linear models. $J$ Roy Stat Soc (B) 73: 3-36.

Yahav, S.; Straschnow, A.; Luger, D.; Shinder, D.; Tanny, J. and Cohen, S. 2004. Ventilation, sensible heat loss, broiler energy, and water balance under harsh environmental conditions. Poultry Sci, 83: 253-258.

Yan, P.S. and Yamamoto, S. 2000. Relationship between thermoregulatory responses and heat loss in piglets. Anim Sci J, 71: 505-509. 